\title{
哌嗪季铵碱催化剂的合成及其在异氰酸酯聚合反应中的应用
}

\author{
许昕䑣 $a$ \\ 谭伟民*,b \\ 姬梦圆 ${ }^{a}$ \\ 杨悦 ${ }^{a}$ \\ 饶兴兴 $b$ \\ 雒新亮 $b$ \\ 张延华 $*, a$ \\ 陈虹宇 $a$ \\ ( ${ }^{a}$ 南京工业大学 化学与分子工程学院 先进化学制造研究院 南京 211816) \\ ( $b$ 国家涂料工程技术研究中心 中海油常州涂料化工研究院有限公司 常州 213016)
}

\begin{abstract}
摘要 在温和条件下合成了一种用于脂肪族异氰酸酯聚合反应的哌嗪类双季铵碱催化剂, 制备过程简单, 且催化剂具 有较高的催化活性和选择性, 催化反应所生成的聚合产物中所需的三聚体成分较高, 产物色度较低.

关键词＼cjkstart季铵碱类催化剂; 合成; 五亚甲基二异氧酸酯; 三聚体
\end{abstract}

\section{Synthesis of Piperazine Quaternary Ammonium Alkali Catalyst and Its Application in Isocyanate Polymerization}

\author{
Xintong $\mathrm{Xu}^{a} \quad$ Weimin Tan*b Mengyuan $\mathrm{Ji}^{a} \quad$ Yue Yang $^{a}$ \\ Xingxing Rao ${ }^{b} \quad$ Xinliang Luo $^{b} \quad$ Yanhua Zhang*, $^{*} \quad$ Hongyu Chen $^{a}$ \\ ( ${ }^{a}$ Institute of Advanced Synthesis, and School of Chemistry and Molecular Engineering, \\ Nanjing Tech University, Nanjing 211816, China) \\ $\left({ }^{b}\right.$ National Engineering Research Center for Coatings, CNOOC Changzhou Paint and \\ Coating Industry Research Institute Co., Ltd., Changzhou, Jiangsu 213016, China)
}

\begin{abstract}
Aliphatic isocyanate trimer has the advantage of excellent thermal stability, radiation resistance, corrosion resistance and flame retardancy. It is an important unit for various chemical products. Hexamethylene diisocyanate trimer (HDIT) is one of the popular trimers, which is mainly synthesized through the polymerization of monomers. A variety of catalysts have been applied in this procedure, including metal salts, phosphines, aminosilanes, $N$-heterocyclic carbenes and $\mathrm{N}$-heterocyclic olefins. However, HDI monomer is usually expensive, while low catalyst activity, harsh preparation conditions, and easy-formation of polymer are often observed in the polymerization process. Pentamethylene diisocyanate (PDI), a cheap bio-based linear aliphatic polyisocyanate with similar structure to traditional HDI, has the potential to replace HDI. Since the activity of PDI is lower, it is found that the catalysts applicable for HDI work not well for PDI. Herein, we design and synthesize 1,4-bis(2-hydroxy-isopropyl)-1,4-dimethylpiperazine bisquaternary ammonium base (PQ-OH) as the catalyst for the production of both HDI trimer and PDI trimer (PDIT). Due to having two hydroxide ions, PQ-OH catalyst can attack the - $\mathrm{NCO}$ groups of two monomers at the same time, thus improving the reaction efficiency. Moreover, the $\beta$-OH groups on the catalyst molecule also promote the polymerization process. The piperazine ring structure in the catalyst has obvious steric hindrance effect, which can enhance the reaction between small molecules, and inhibit the reaction between large molecules and small molecules. Thus, the product with high trimer content is obtained. A general synthesis procedure for PQ-OH is described as followed. Propylene epoxide $(70.0 \mathrm{~mL}, 1.0 \mathrm{~mol})$ is added to a mixture of 1,4-dimethylpiperazine $(67.6 \mathrm{~mL}, 0.5$ $\mathrm{mol})$ and water $(18 \mathrm{~mL}, 1.0 \mathrm{~mol})$ dropwisely under argon. After stirring at room temperature for $4 \mathrm{~h}$, the solution turns to brown and stratifies. The lower layer is separated and concentrated in vacua to remove excess propylene oxide, water and possible alcohol by-products. The obtained crude product is dissolved in methanol, followed by decolorizing with activated carbon and drying with molecular sieve. After filtering and concentration, the desired product is obtained as a white viscous liquid.
\end{abstract}

Keywords quaternary ammonium catalyst; synthesis; pentamethylene diisocyanate; trimer

\section{1 引言}

聚氨酯是一类用途非常广泛的聚合物, 其中, 由异 氰酸酯单体聚合产生的三聚体是比较重要的聚氨酯之
一, 具有较强的化学稳定性和物理机械性能. 特别是脂 肪族异氰酸酯三聚体, 由于其在热稳定性、耐辐射性、 耐黄变性、耐腐蚀性以及阻燃性等方面表现优异, 已被 广泛应用于高档油漆和高级涂料等化工产品的生产 ${ }^{[1]}$.

\footnotetext{
*E-mail: tanwm@cnooc.com.cn; ias_yhzhang@njtech.edu.cn

Received June 29, 2021; published July 30, 2021.

Supporting information for this article is available free of charge via the Internet at http://sioc-journal.cn.

Project supported by the National Natural Science Foundation of China (Nos. 21673117, 91956109), Jiangsu Provincial Foundation for Specially-Appointed Professor, and Start-up Fund from Nanjing Tech University (Nos. 39837126, 39837102).

项目受国家自然科学基金(Nos. 21673117, 91956109)、江苏省特聘教授基金和南京工业大学科研启动基金(Nos. 39837126, 39837102)资助.
} 
因此, 高效且绿色环保的脂肪族异氰酸酯三聚体的合成 方法的研究具有比较重要的实用价值.

目前脂肪族异氰酸酯三聚体的合成主要采用本体 聚合的方法 ${ }^{[2]}$. 由于异氰酸酯的-NCO 基团中, 碳原子 上的电子云密度较小, 显正电, 容易受到亲核试剂的进 攻，所以在催化剂作用下，分子间的-NCO 基团会相互 加成, 从而发生聚合反应 ${ }^{[3]}$. 受到催化剂及反应条件的 影响, 多聚体的生成是不可避免的, 但从应用的角度出 发, 一定量的多聚体对于提升产品的干性和刚性是有利 的, 因而国内外的相关合成研究中, 基本不涉及除去高 聚物, 而是予以保留. 报道中所说的三聚体产物也不是 纯的三聚体, 而是含有一定比例三聚体的混合物. 为了 提高聚合反应的效率和选择性, 人们尝试了多种类型的 催化剂, 例如金属盐催化剂[4]、有机膦催化剂[5]、有机硅 催化剂 ${ }^{[6]}$ 、氮杂环卡宾 ${ }^{[7]}$ 以及氮杂环烯烃 ${ }^{[8]}$ 等. 但是, 这 些催化剂存在活性较低、易生成多聚产物、反应条件苛 刻或者催化剂本身有毒性等问题. 相较之下, 季铵盐类 和季铵碱类催化剂表现出较好的性能, 具有低毒性、低 成本和稳定性好等特点. 例如, Hsieh 等[9]用三甲基-2-羟 丙基-2-乙基己酸铵为催化剂, 成功合成出六亚甲基二 异氰酸酯三聚体(HDIT)为主要产物的聚合物, 反应条件 温和且容易控制; 然而, 该催化剂制备过程复杂, 多聚 体含量较高. 谭伟民等 ${ }^{[10]}$ 以 2-羟丙基三甲基辛酸铵盐 (TMR)作为催化剂, 成功催化了活性相对较低的五亚甲 基二异氰酸酯(PDI)的三聚反应, 制得浅黄色透明的五 亚甲基二异氰酸酯三聚体(PDIT). 在该反应中, 异氰酸 酯单体可受到催化剂所含 $\beta-\mathrm{OH}$ 基团的亲核进攻生成具 有助催化作用的氨基甲酸酯. 助催化的机理可能是异氰 酸酯-NCO 中的 $\mathrm{O}$ 和生成的氨基甲酸酯中的氨基 $\mathrm{H}$ 之间 会形成氢键, 使 NCO 基团中碳原子的极化程度增大, 亲电性增加, 进而加速了聚合反应的进程 ${ }^{[2]}$; 然而该催 化剂在反应过程中会导致体系变黄, 影响三聚体产品的 色度与外观, 不利于后续在聚氨酯涂料或油漆中的应 用. 相比上述季铵盐类催化剂而言, 季铵碱类催化剂本 身是较强的亲核试剂, 更易与-NCO 作用, 因此其催化 活性会更好一些，例如，邱少君等 ${ }^{[11]}$ 使用三甲基羟乙基 氢氧化铵作为催化剂, 成功得到了 HDIT 含量高达 $63 \%$ 的低色度产物. 然而, 已报道的季铵碱类催化剂的制备 方法较为繁琐，一般通过季铵盐加碱的方式制得. 因此, 如何绿色高效地制备具有较高催化活性和选择性的季 铵碱类催化剂, 对脂肪族异氰酸酯的三聚体合成反应具 有重要意义.

HDIT 是脂肪族二异氰酸酯三聚体中的主流产品, 但是随着对可持续发展需求的增加, HDI 原料供给、环 境、经济因素等问题日益凸显, 造成供需关系失衡, 给 高端聚氨酯涂料用户带来巨大的成本压力. 因此, 发展 新型 “绿色涂料” 势在必行 ${ }^{[12]}$. 以生物质原料合成的 PDI, 其结构与 HDI 高度相似, 具备替代 HDI 的理论可
行性，所得 PDIT 有望用于聚氨酯涂料，替代传统固化 剂, 实现绿色可持续发展 ${ }^{[13]}$. 但是, 由于 PDI 活性较低, 所报道的用于 PDI 聚合反应的催化剂与 HDI 相比少之 又少. 这就为新的催化剂的设计合成提出了需求.

本工作以 1,4-二甲基哌嗪、环氧丙烷和水为原料, 在温和条件下成功制备出了 1,4-二(2-羟基异丙基)-1,4二甲基哌嗪双季铵碱(PQ-OH)这种既可用于 HDI, 亦可 用于 PDI 聚合反应的催化剂. 该催化剂由于含有两个氢 氧根，可同时进攻两个单体的- $\mathrm{NCO}$ 基团，从而提高了 反应效率. 而且催化剂所含的 $\beta-\mathrm{OH}$ 基团也对聚合反应 有一定的促进作用. 催化剂中引入的哌嗪环状结构, 具 有明显的位阻效应，可以促进小分子之间的反应，并抑 制大分子与小分子或大分子之间的反应，从而得到三聚 体含量较高的产品.

\section{2 结果与讨论}

\section{$2.1 \mathrm{PQ}-\mathrm{OH}$ 季铵碱催化剂的制备}

参考陈卓 ${ }^{[2]}$ 合成季铵碱的方法，我们以 1,4-二甲基 哌嗪、环氧丙烷和水作为原料, 按一定比例混合后, 在 氩气保护下室温搅拌一定时间. 然后, 将反应混合物分 液, 所得下层粗产物经乙酸乙酯洗涤后浓缩干燥, 得到 的深褐色粘稠状液体, 即为哌嗪季铵碱催化剂, 该催化 剂可直接用于催化聚合反应.

为了探究所制备的催化剂的催化效率和选择性, 我 们将其应用于生物质原料合成的 PDI 聚合反应中. 按照 通用的合成方法, 我们待 PDI 单体稳定在 $80{ }^{\circ} \mathrm{C}$ 后, 向 其中加入自制的催化剂进行反应, 当 $\mathrm{NCO}$ 含量降至 $40 \%(w)$ 时, 加入阻聚剂, 降温出料, 所生成的聚合物色 度按照 GB/T 3143-1982 方法测定(表 1). 通过调节制备 催化剂时的原料比例和反应时间，我们可以确定催化效 果较好的催化剂的制备条件. 实验结果表明, 当原料比 例一定时，随着制备时间的延长(表 1, Entries 1，2)，所 得催化剂应用于 PDI 聚合反应后, 催化效率有所降低, 产物色度也明显变高. 当制备时间一定时, 改变反应物 中环氧丙烷(表 1, Entries 1,3，4)或水(表 1, Entries 3, 5, 6) 的比例, 所制催化剂用于催化 PDI 聚合反应后的产物色 度会有一个最低值. 通过比较分析, 我们发现一个比较 好的制备催化剂的条件是控制 1,4-二甲基哌嗪、水和环 氧丙烷的物质的量比为 $1: 2: 2$, 反应时间为 $4 \mathrm{~h}$, 此时 所得的催化剂催化效率较高, 且聚合反应产物色度低于 $45 \mathrm{~Hz}$ (表 1, Entry 3). 值得一提的是, 在制备催化剂的后 处理过程中, 加入活性炭不仅可以使催化剂脱色, 还可 以去除催化剂中可能的杂质和副产物(表 1, Entry 7). 在 最优的合成条件下，加入活性炭脱色纯化后，可以得到 白色粘稠液体状的催化剂(PQ-OH), 纯度可达 $98.7 \%$, 产率为 $20.6 \%$, 最大限度地降低了其对后续聚合反应的 色度影响. 
表 1 反应条件优化

Table 1 Optimization of the reaction conditions

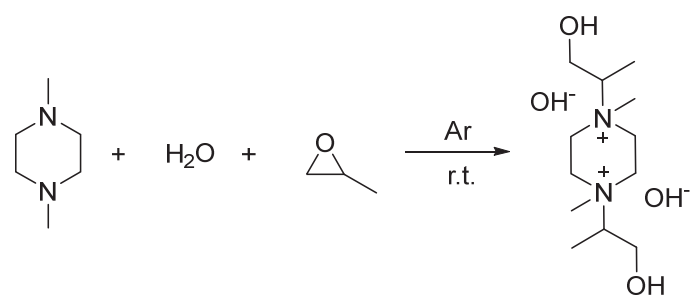

$\mathrm{PQ}-\mathrm{OH}$

\begin{tabular}{ccccc}
\hline Entry $^{a}$ & Ratio $^{b}$ & $t^{c} / \mathrm{h}$ & $t^{d} / \mathrm{h}$ & Chroma/Hz \\
\hline 1 & $1: 2: 1.5$ & 4 & 0.75 & $<70$ \\
2 & $1: 2: 1.5$ & 9 & 1 & $<90$ \\
3 & $1: 2: 2$ & 4 & 1.5 & $<45$ \\
4 & $1: 2: 2.5$ & 4 & 4.2 & $<60$ \\
5 & $1: 1.5: 2$ & 4 & 5.5 & $<60$ \\
6 & $1: 2.5: 2$ & 4 & 1.25 & $<60$ \\
7 & $1: 2: 2$ & 4 & 1.25 & $<45$ \\
\hline
\end{tabular}

${ }^{a}$ Reaction conditions: substrates were stirred at room temperature in an argon atmosphere. ${ }^{b}$ Ratio $=n(1,4$-dimethylpiperazine $): n\left(\mathrm{H}_{2} \mathrm{O}\right): n$ (propylene oxide). ${ }^{c}$ Time to prepare catalyst. ${ }^{d}$ Time of polymerization reaction.

\section{$2.2 \mathrm{PQ}-\mathrm{OH}$ 季铵碱催化剂在 PDI 聚合反应中的应用}

\subsubsection{PDI 三聚体的合成}

为了进一步优化所制备的 PQ-OH 催化剂对 PDI 三 聚反应的催化效果, 我们对催化剂的用量、聚合反应的 温度和时间进行了探讨, 结果总结在表 2 中. 当反应温 度为 $80{ }^{\circ} \mathrm{C}$ 时, 我们发现如果催化剂的用量过少, 则聚 合反应根本不发生(表 2, Entry 1). 随着催化剂用量的增 加, PDI 单体的转化率和三聚体的含量都有所增加(表 2, Entries 2 4), 其中产物色度比较低时的催化剂加入量

表 2 PDI 三聚反应条件优化

Table 2 Optimization of PDI trimerization reaction conditions

$$
\text { NCO }
$$

\begin{tabular}{ccccccc}
\hline Entry $^{a}$ & $\mathrm{PQ}-\mathrm{OH} / \%$ & $T /{ }^{\circ} \mathrm{C}$ & $t / \mathrm{h}$ & Conv. $^{b} / \%$ & PDIT $^{b} / \%$ & Chroma $/ \mathrm{Hz}$ \\
\hline 1 & 0.04 & 80 & - & - & - & - \\
2 & 0.05 & 80 & 4 & 74.8 & 45.4 & $<60$ \\
3 & 0.06 & 80 & 1.3 & 75.1 & 48.1 & $<45$ \\
4 & 0.07 & 80 & 0.5 & 80.0 & 56.4 & $<60$ \\
5 & 0.06 & 70 & 4 & 63.3 & 39.5 & $<45$ \\
6 & 0.06 & 90 & 0.5 & 84.1 & 51.3 & $<60$ \\
7 & 0.06 & 80 & 1 & 73.7 & 46.9 & $<45$ \\
8 & 0.06 & 80 & 2 & 78.4 & 54.1 & $<45$ \\
9 & 0.06 & 80 & 3 & 81.2 & 48.3 & $<45$ \\
10 & 0.06 & 80 & 4 & 82.3 & 43.7 & $<45$ \\
\hline
\end{tabular}

${ }^{a}$ Reaction conditions: Preheat PDI $(20 \mathrm{~g})$ to $90{ }^{\circ} \mathrm{C}$ for $1 \mathrm{~h}$ in an argon atmosphere, then reduce the temperature to $T{ }^{\circ} \mathrm{C}$ and add the catalyst. ${ }^{b}$ Based on HPLC analysis.
为 PDI 单体质量的 $0.06 \%$. 聚合反应的温度对产物的影 响是比较明显的，这是由于该反应本身是放热反应，随 着反应温度的升高, 聚合反应速率明显加快, 但三聚体 产物含量也随之降低, 更趋向于生成多聚产物(表 2 , Entries 3, 5, 6). 除此之外, 反应时间也是聚合反应程度 的重要影响因素. 随着反应时间的延长, PDI 单体的转 化率有所增加(表 2, Entries 7 10), 但产物中 PDI 三聚 体的含量是有一个峰值的(表 2, Entry 8), 这说明反应产 生的三聚体会在催化剂的作用下继续发生聚合反应生 成高聚物, 所以我们需要选取一个比较合适的反应时 间. 实验结果表明, 当在 $80{ }^{\circ} \mathrm{C}$ 加入 PDI 单体质量的 $0.06 \%$ 催化剂时, 反应 $2 \mathrm{~h}$ 是比较适宜的, PDI 单体的转 化率可达 $78.4 \%$, 所得聚合物中三聚体含量可达 $54.1 \%$, 色度低于 $45 \mathrm{~Hz}$ (表 2, Entry 8).

随后，我们将自制 PQ-OH 催化剂用于 HDI 的聚合 反应, 发现当反应温度为 $80{ }^{\circ} \mathrm{C}$, 催化剂用量为 PDI 单 体质量的 $0.04 \%$, 反应 $1 \mathrm{~h}$ 时 HDI 单体的转化率可达 $52.1 \%$, 所得聚合物中三聚体含量可达 $44.2 \%$, 色度低于 $35 \mathrm{~Hz}$, 与已报道的催化剂相比 ${ }^{[2]}$, 效果更佳. 之后, 为 了进一步评估 PQ-OH 的活性, 我们选择了市售的 2-羟 丙基三甲基辛酸铵盐(TMR-4)与芐基三甲基氢氧化铵 (BHS)这两种常见的用于脂肪族异氰酸酯聚合反应的催 化剂, 来进行 PDI 的聚合反应(表 3). 由实验结果可知, 在同样的反应条件下, BHS 无法催化 PDI 的聚合反应(表 3 , Entry 1), 这可能是由于 PDI 单体相较于 HDI 单体而 言反应活性较低. 而含有 $\beta$-OH 的 TMR-4 催化剂虽然对 PDI 的聚合反应具有一定的催化效果(表 3, Entry 2), 但 无论是单体转化率, 还是三聚体含量, 都低于自制的 PQ-OH(表 3, Entry 3).

表 3 不同催化剂催化活性对比

Table 3 Catalytic activity of different catalysts

\begin{tabular}{cccccc}
\hline Entry $^{a}$ & Catalyst $^{b}$ & $t / \mathrm{h}$ & Conv. $^{b} \%$ & PDIT $^{b} \%$ & Chroma/Hz \\
\hline 1 & BHS & - & - & - & - \\
2 & TMR-4 & 2 & 66.67 & 49.24 & $<45$ \\
3 & PQ-OH & 2 & 78.44 & 54.14 & $<45$ \\
\hline
\end{tabular}

${ }^{a}$ Reaction conditions: Preheat PDI $(20 \mathrm{~g})$ to $90{ }^{\circ} \mathrm{C}$ for $1 \mathrm{~h}$ in an argon atmosphere, then reduce the temperature to $T{ }^{\circ} \mathrm{C}$ and add the catalyst. ${ }^{b}$ Based on HPLC analysis.

\subsubsection{PDI 三聚体的红外光谱(FTIR)分析}

为了探究所得聚合产物的结构, 我们对其进行了红 外光谱分析(图 1). $2943 \mathrm{~cm}^{-1}$ 处为脂肪族的亚甲基吸收 峰, 在 $2260 \mathrm{~cm}^{-1}$ 处的强烈吸收峰为异氧酸酯基- $\mathrm{NCO}$ 的 伸缩振动吸收峰, $582 \mathrm{~cm}^{-1}$ 处为- $\mathrm{NCO}$ 的变形振动峰. 1681、1460 和 $765 \mathrm{~cm}^{-1}$ 处的吸收峰是异氧腿酸酯环的 特征吸收峰, 这组特征峰的出现说明产物中存在异氧腿 酸酯环结构. 通过对比可知, PDI 三聚体在 1681、1460、 $765 \mathrm{~cm}^{-1}$ 处出现明显的吸收峰, 说明异氧酸酯发生了自 聚, 生成了环状的三聚体甚至多聚体结构. 


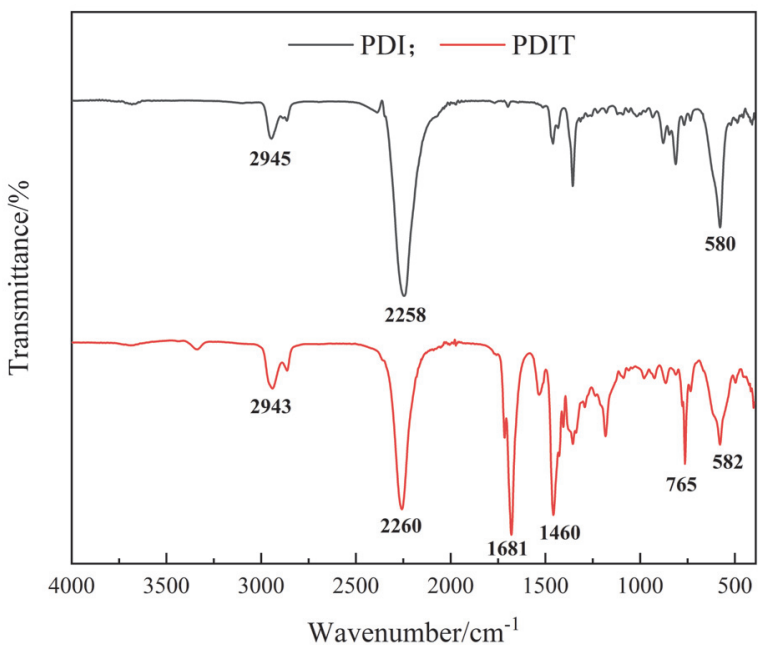

图 1 PDI 单体与三聚体产物的红外光谱

Figure 1 Infrared spectra of PDI and PDIT

\subsubsection{PDI 三聚体的凝胶渗透色谱 (GPC)分析}

红外光谱仅能证实异氰腿酸酯环和主要官能团的 存在, 不能直接证明三聚体的存在, 需结合 GPC 进一步 确认.

GPC 是根据聚合物的分子量大小来进行分离的, 相对分子质量越大, 淋出时间越短. 对于不同组分分子 量的确定, GPC 的分析方法是先用标样做一条相对分子 质量与淋出时间的对应关系曲线(即校正曲线), 然后根 据样品的淋出时间来确定其分子量. 可见此方法所得的 分子量是相对于标样的, 有时可能存在较大偏差, 因此 需要同时结合样品淋出时间来判定. 可用的聚合物标样 的种类并不多, 我们利用常用的聚苯乙烯作为标样, 将 PDI 单体、市售 PDI 三聚体 D3725 和 PQ-OH 催化生成 的 PDI 聚合物进行 GPC 分析(图 2). 结合淋出时间和分 子量结果推断淋出时间为 $9.6 \mathrm{~min}$, 峰位相对分子质量 $\left(M_{\mathrm{p}}\right)$ 为 538 的是 $\mathrm{PDI}$ 三聚体; 出峰时间为 $10.3 \mathrm{~min}, M_{\mathrm{p}}$

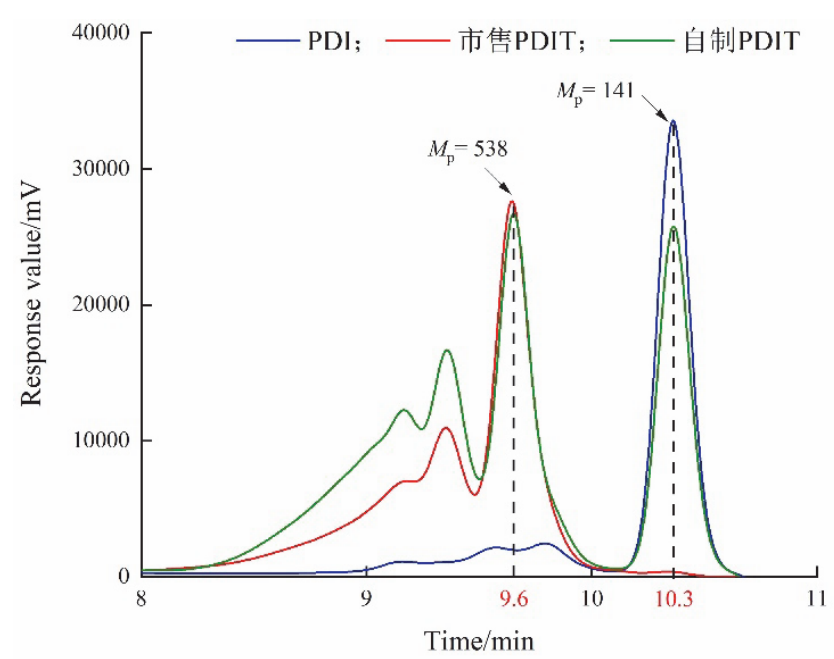

图 2 PDI 三聚体与 PDI 的凝胶渗透色谱

Figure 2 Gel permeation chromatography of PDIT and PDI
为 141 的是 PDI 单体. 由图中可以看出, PQ-OH 催化生 成的 PDI 聚合物中(图 2, 绿色曲线), 除了三聚体和未反 应的单体外, 在 $9.6 \mathrm{~min}$ 和 $10.3 \mathrm{~min}$ 之间并未出现其他 峰, 说明产物中基本不存在二聚体. 除此之外, 保留时 间 $<9.2 \mathrm{~min}$ 的部分存在一个拖尾峰, 说明有少量高聚 物生成.

\section{3 可能的机理}

异氰酸酯的 $-\mathrm{NCO}$ 基团中, 氮和氧原子上的电子云 密度较大, 而碳原子上的电子云密度较小, 因此, 结合 已报道的异氧酸酯催化聚合反应的机理 ${ }^{[14-15]}$, 我们推测 PQ-OH 催化剂催化的 PDI 聚合反应的机理如下(图 3). 首先, $\mathrm{PQ}-\mathrm{OH}$ 催化剂的两个 $\mathrm{OH}^{-}$分别与两个 $\mathrm{PDI}$ 单体分 子中的-NCO 基团反应，得到中间体 INT1; 随后，该中 间体中的阴离子部分作为亲核试剂继续进攻第三个 PDI 单体分子的-NCO 基团, 最后释放催化剂, 得到 PDI 三 聚体(Path A). 多聚体是在三聚体的基础上，中间体进 一步与更多的 PDI 单体反应生成. 另一方面, 由于中间 体 INT1 的亲核性，使其具有同时作用于两个 PDI 单体 分子, 生成二聚体(PDID)的可能(Path B). 两种途径为 竞争反应, 然而反应结果中未检测到二聚体的存在, 推 测反应途径 B 可能所需能垒更高, 所以不易发生.

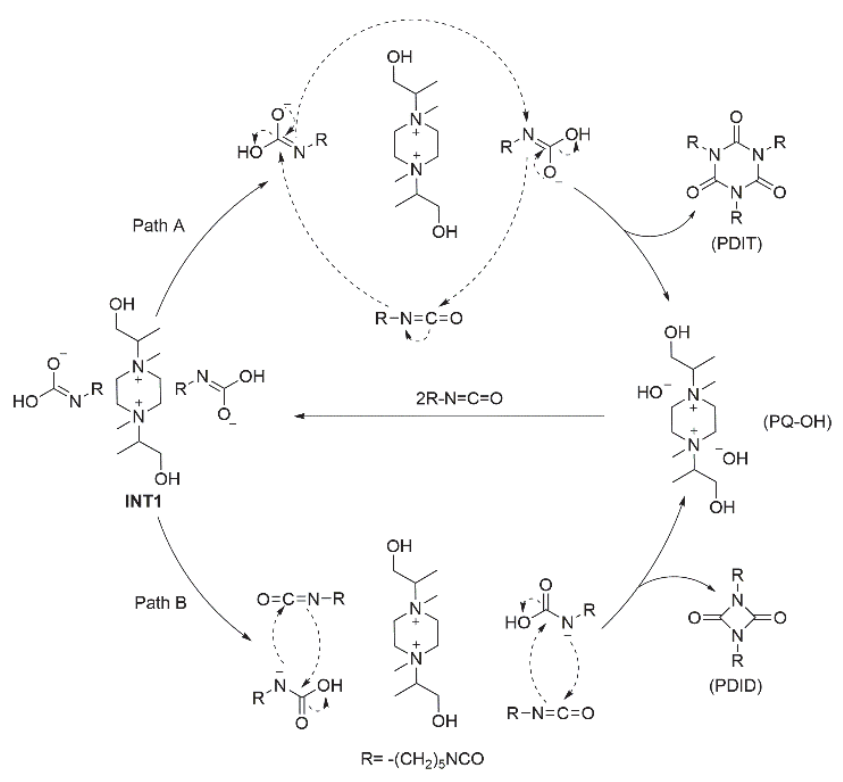

图 3 可能的反应机理

Figure 3 Proposed reaction mechanism

\section{3 结论}

本工作设计并合成了含有 $\beta-\mathrm{OH}$ 的哌嗪季铵碱类催 化剂，其制备条件温和，原料廉价易得，纯化过程简单. 实验结果表明, 合成催化效果较好的催化剂的条件是 1,4-二甲基哌嗪、水和环氧丙烷的物质的量比为 $1: 2$ : 2 , 反应时间为 $4 \mathrm{~h}$. 经过纯化处理, 可以得到白色粘稠 状液体催化剂. 将 PDI 单体质量的 $0.06 \%$ 的催化剂用于 
PDI 的聚合反应中时, 在 $80{ }^{\circ} \mathrm{C}$ 下反应 $2 \mathrm{~h}$, 就可得到 PDI 三聚体含量为 $54.1 \%$ 的聚合产物, 且色度低于 45 Hz. 与市售的季铵型催化剂相比, 我们所制备的催化剂 性能更为优越. 同时, 我们根据实验结果和活性中间体 的结构特征, 分析并阐明了可能的催化反应机理. 本工 作对于设计并合成用于异氰酸酯聚合反应的催化剂具 有很好的指导意义.

\section{4 实验部分}

\section{1 反滴定法}

用 $10 \mathrm{~mL}$ 移液管移取 $0.5 \mathrm{~mol} / \mathrm{L}$ 二正丁胺-甲苯溶液, 加入到 $0.12 \mathrm{~g}$ 试样中, 摇匀, 用磁力搅拌 5 $10 \mathrm{~min}$ 后加 入 $3 \sim 4$ 滴溴甲酚绿指示剂, 此时溶液为蓝绿色. 以标定 好的实际浓度为 $0.15 \mathrm{~mol} / \mathrm{L}$ 盐酸-无水乙醇标准溶液滴 定至蓝色刚好转变为浅黄色(过渡状态为黄绿色), 且 1 $\min$ 不变色, 此时为滴定终点, 记下此时消耗盐酸标准 滴定溶液的体积 $V_{2}(\mathrm{~mL})$. 进行空白试验, 消耗的盐酸 标准滴定溶液的体积为空白体积 $V_{1}(\mathrm{~mL})$, 计算公式如 下:

$$
N C O=\frac{\left(V_{1}-V_{2}\right) c \times 4.2}{m}=\left(V_{1}-V_{2}\right) \times 5.25
$$

\section{2 哌嗪类季铵碱的制备方法}

在氩气保护下, 将环氧丙烷 $(70.0 \mathrm{~mL}, 1.0 \mathrm{~mol})$ 缓慢 滴加到 1,4-二甲基哌嗪(67.6 mL, $0.5 \mathrm{~mol}$ )与水 $(18 \mathrm{~mL}$, $1.0 \mathrm{~mol}$ )的混合溶液中. 然后在室温下搅拌 $4 \mathrm{~h}$. 反应结 束后溶液变为褐色, 且有分层. 上层液体为未反应完全 的原料, 可回收再次利用. 下层液体先用乙酸乙酯洗涤 至无有色物质洗出, 再通过旋蒸除去多余的环氧丙烷、 水以及可能生成的醇类副产物, 得到深褐色的粘稠状液 体, 即为哌嗪季铵碱催化剂粗品, 产率大约为 $46.3 \%$ 左 右, 可直接用于催化聚合反应. 为了降低催化剂的颜色 对产物色度的影响, 我们对催化剂进行进一步纯化. 每 $1 \mathrm{~g}$ 粗产物用 $10 \mathrm{~mL}$ 甲醇进行稀释, 然后加入 $4 \mathrm{~g}$ 活性炭 搅拌进行脱色. 过滤后, 再加入分子篎除去可能存在的 水. 最后得到无色滤液, 经旋蒸浓缩后得到白色粘稠状 液体, 即为目标产物 PQ-OH. ${ }^{1} \mathrm{H}$ NMR $\left(\mathrm{D}_{2} \mathrm{O}, 400 \mathrm{MHz}\right)$ $\delta$ : $1.14(\mathrm{dd}, J=53.4,6.6 \mathrm{~Hz}, 5 \mathrm{H}), 1.85$ (s, 1H), 2.31 (s, $3 \mathrm{H}), 2.81(\mathrm{~s}, 4 \mathrm{H}), 3.18 \sim 3.54(\mathrm{~m}, 14 \mathrm{H}), 4.42(\mathrm{~s}, 1 \mathrm{H}) ;{ }^{13} \mathrm{C}$ NMR $\left(\mathrm{D}_{2} \mathrm{O}, 400 \mathrm{MHz}\right) \delta: 18.1,21.2,23.4,43.4,47.5,48.9$, 61.0, 61.8, 66.6, 67.9, 160.7, 181.7; HRMS (ESI ${ }^{+}$): Exact mass calcd for $\mathrm{C}_{12} \mathrm{H}_{30} \mathrm{~N}_{2} \mathrm{O}_{4}, \quad\left[\mathrm{M}+\mathrm{Na}^{+}\right]$requires $\mathrm{m} / z$ 289.2098 found 289.2484.

\subsection{PDI 聚合反应}

将 PDI 单体 $(0.13 \mathrm{~mol}, 20 \mathrm{~g})$ 加入反应瓶中, 在氩气 保护下升温至 $90{ }^{\circ} \mathrm{C}$, 预热 $1 \mathrm{~h}$ 后降温至特定温度, 待温 度稳定后, 滴加 PQ-OH 催化剂溶液(采用甲醇进行稀释, 稀释度为 $10 \%$ ), 滴加速度为 1 滴/10 $20 \mathrm{~s}$, 控制反应温 度浮动范围不超过 $\pm 1{ }^{\circ} \mathrm{C}$. 用反滴定法确定体系中 $\mathrm{NCO}$ 的含量, 以此来监测反应进度. 当 NCO 含量达到 要求后, 加入磷酸二丁酯阻聚剂, 降温出料. 反应结束 后, 未反应的 PDI 单体在 $140{ }^{\circ} \mathrm{C}$ 油浴下, 用薄膜蒸发器 减压(100 Pa 以下)蒸馏除去. 由于反应体系中季铵碱催 化剂的用量仅为反应物质量的万分之六，对最终产物性 质基本无影响，所以无需处理除去.

\section{References}

[1] (a) He, Y.; Zhang, X.; Zhang, X.; Huang, H.; Chang, J.; Chen, H. J. Ind. Eng. Chem. 2012, 18, 1620; (b) Giuglio-Tonolo, A. G.; Spitz, C.; Terme, T.; Vanelle, P. Tetrahedron Lett. 2014, 55, 2700; (c) Driest, P. J.; Lenzi, V.; Marques, L. S. A.; Ramos, M. M. D.; Dijkstra, D. J.; Richter, F. U.; Stamatialis, D.; Grijpma, D. W. Polym. Adv. Technol. 2017, 28, 1299; (d) Wu, L.; Liu, W.; Ye, J.; Cheng, R. Catal. Commun. 2020, 145, 106097.

[2] Chen, Z. M.S. Thesis, South China University of Technology, Guangzhou, 2014 (in Chinese). (陈卓, 硕士论文, 华南理工大学, 广州, 2014.)

[3] Qiu, S. J.; Gan, X. X.; Lu, X. M. Chem. Adhesion 2001, 04, 165 (in Chinese). (邱少君, 甘孝贤, 卢先明, 化学与粘合, 2001, 04, 165.)

[4] Sharpe, H. R.; Geer, A. M.; Williams, H. E.; Blundell, T. J.; Lewis, W.; Blake, A. J.; Kays, D. L. Chem. Commun. 2017, 53, 937.

[5] Helberg, J.; Oe, Y.; Zipse, H. Chemistry 2018, 24, 14387.

[6] Roman, M.; Andrioletti, B.; Lemaire, M.; Bernard, J.-M.; Schwartz, J.; Barbeau, P. Tetrahedron 2011, 67, 1506.

[7] Duong, H. A.; Cross, M. J.; Louie, J. Org. Lett. 2004, 6, 4679.

[8] Li, C.; Zhao, W.; He, J.; Zhang, Y. Chem. Commun. 2019, 55, 12563.

[9] Hsieh, K. H.; Kresta, J. E. In ACS Symposium Series, Vol. 195, Ed. George, B.; Butler, E., Kresta, America, 1982, Chapter 24, p. 311.

[10] Tan, W. M.; Wang, L.; Di, Z. G.; Luo, X. L.; Shi, L. P.; Wang, Y. X.; Yu, F.; Xu, X.; Chen, K. Q. PCI 2020, 50, 38 (in Chinese). (谭伟民, 王黎, 狄志刚, 雒新亮, 史立平, 王亚金鍂, 郁飞, 许旭, 陈可泉, 涂料工业, 2020, 50, 38.)

[11] Qiu, S. J.; Gan, X. X.; Wang, J. G.; Yang, Y. PUI 1998, 02, 17 (in Chinese). (邱少君, 甘孝贤, 王吉贵, 杨毅, 聚氨酯工业, 1998, 02, 17.)

[12] Luo, X. L.; Di, Z. G.; Tan, W. M.; Shi, L. P.; Wang, L.; Wang, Y. X. PCI 2020, 50, 1 (in Chinese). (雒新亮, 狄志刚, 谭伟民, 史立平, 王黎，王亚金金，涂料工业, 2020, 50, 1.)

[13] Morales-Cerrada, R.; Tavernier, R.; Caillol, S. Polymers 2021, 13, 1255.

[14] Siebert, M.; Sure, R.; Deglmann, P.; Closs, A. C.; Lucas, F.; Trapp, O. J. Org. Chem. 2020, $85,8553$.

[15] Peters, S. J.; Klen, J. R.; Smart, N. S. Org. Lett. 2008, 10, 4521. 